\title{
The psychology of "swiping": A cluster analysis of the mobile dating app Tinder
}

\author{
LUCIEN ROCHAT ${ }^{1}$, FRANCESCO BIANCHI-DEMICHELI ${ }^{1,2,3}$, ELIAS ABOUJAOUDE ${ }^{4}$ and YASSER KHAZAAL ${ }^{5,6,7}$ \\ ${ }^{1}$ Faculty of Psychology and Educational Sciences, University of Geneva, Geneva, Switzerland \\ ${ }^{2}$ Faculty of Medicine, University of Geneva, Geneva, Switzerland \\ ${ }^{3}$ Department of Obstetrics and Gynecology, University Hospitals of Geneva, Geneva, Switzerland \\ ${ }^{4}$ Department of Psychiatry and Behavioral Sciences, Stanford University School of Medicine, Stanford, CA, USA \\ ${ }^{5}$ Addiction Medicine, Department of Psychiatry, Lausanne University Hospital, Lausanne, Switzerland \\ ${ }^{6}$ Research Centre, University Institute of Mental Health at Montreal, Montreal, Canada \\ ${ }^{7}$ Faculty of Biology and Medicine, Lausanne University, Lausanne, Switzerland
}

(Received: April 24, 2019; revised manuscript received: September 4, 2019; accepted: September 29, 2019)

\begin{abstract}
Background and aims: The use of the smartphone dating application Tinder is increasingly popular and has received much media attention. However, no empirical study to date has investigated the psychological characteristics driving its adaptive or problematic use. The aim of this study is to determine whether reliable subtypes of users can be identified via a cluster analysis approach. Methods: A total of 1,159 Tinder users were recruited. Survey questions investigated user characteristics, including: motives for app use, sexual desire, attachment styles, impulsivity traits, self-esteem, problematic use, depressive mood, and patterns of use. Results: Four reliable clusters were identified: two with low levels of problematic use ("regulated" and "regulated with low sexual desire"), one with an intermediate level of problematic use ("unregulated-avoidants"), and one with a high level of problematic use ("unregulated-highly motivated"). The clusters differed on gender, marital status, depressive mood, and use patterns. Conclusion: The findings provide insight into the dynamic relationships among key use-related factors and shed light on the mechanisms underlying the self-regulation difficulties that appear to characterize problematic Tinder use.
\end{abstract}

Keywords: Tinder, cybersex, impulsivity, attachment, self-esteem, motives

\section{INTRODUCTION}

Launched in 2012, the mobile dating application (app) Tinder has quickly gained popularity and currently counts over 50 million users worldwide (Smith, 2018). In contrast to "traditional" online dating sites, this smartphone app has the advantage of enhanced portability and access, as well as geolocalization capacity (Schrock, 2015). After creating an account that includes photographs, an optional short description and search preferences stratified by age (minimum 18 years), gender, as well as distance, Tinder allows users to quickly and anonymously like ("swipe right") or reject ("swipe left") profiles of other individuals in their geographic vicinity. If two users swipe right on each other's profile, they are "matched" and can chat and potentially meet to pursue a short- or long-term relationship.

Increased access to potential mates, the user-friendly nature of the app, the initial matching provided by the simultaneity of likes, and the ease of finding local - and therefore more readily available - mates can lead to difficulty controlling app use (Orosz et al., 2018), a problem that has caused some authors to approach problematic Tinder use as a behavioral addiction (Orosz, Tóth-Király, Bőthe, \& Melher, 2016).

The complete reconfiguration of the dating and sexual landscape afforded by the Internet (Aboujaoude, 2011) would seem to have been accelerated by mobile apps such as Tinder, raising crucial questions for individuals and society at large. As such, understanding the psychological factors underlying their use is highly important. Although some small studies have examined motives, personality traits, and self-esteem characteristics associated with adaptive Tinder use, a clear understanding of its "problematic" use is still lacking. This study aims to explore the joint influence of psychological factors that have either already been implicated in adaptive or "problematic" Tinder use (e.g., motives and self-esteem), or that, despite not having been formally studied, are likely to be relevant due to their association with other behavioral addictions (e.g., attachment style, impulsivity, and sexual desire; Varfi et al., 2019).

\section{Motives underlying use}

The Uses and Gratifications Theory has been invoked to account for the popularity of Tinder by emphasizing that the app helps meet physical (e.g., sexual pleasure), social

* Corresponding author: Lucien Rochat, PhD; Faculty of Psychology and Educational Sciences, University of Geneva, 40, Boulevard du Pont d'Arve, CH-1205 Geneva, Switzerland; Phone: +41 2237993 46; E-mail: Lucien.Rochat@unige.ch

This is an open-access article distributed under the terms of the Creative Commons Attribution-NonCommercial 4.0 International License, which permits unrestricted use, distribution, and reproduction in any medium for non-commercial purposes, provided the original author and source are credited, a link to the CC License is provided, and changes - if any - are indicated. 
(e.g., finding a romantic partner or friend), and psychosocial needs (e.g., validating one's physical attractiveness; Sumter, Vandenbosch, \& Ligtenberg, 2017; Timmermans \& De Caluwé, 2017). In line with other work on cybersex and gratification-seeking, some studies suggest that Tinder use may constitute a coping mechanism intended to regulate depressive affect (Laier \& Brand, 2014; Sumter et al., 2017) and improve self-esteem (Orosz et al., 2018), with possible gender differences across motives (e.g., more interest in "hooking up" among men; Ranzini \& Lutz, 2017).

\section{Self esteem}

Self-esteem has been defined as the positive or negative attitude toward oneself (Rosenberg, 1965). High self-esteem has been linked to authentic self-representation on Tinder (Ranzini \& Lutz, 2017), whereas low self-esteem has been linked to sexting (sharing sexually explicit photos of oneself; Ybarra \& Mitchell, 2014) and excessive sexual behaviors (Andreassen, Pallesen, Griffiths, Torsheim, \& Sinha, 2018). Still, other studies reported mixed results on the association between Tinder use and self-esteem (e.g., Gatter \& Hodkinson, 2016; Strubel \& Petrie, 2017).

\section{Impulsivity}

Impulsivity refers to actions that are "poorly conceived, prematurely expressed, or unduly risky" (Daruna \& Barnes, 1993, p. 23). It has been considered a defining characteristic of online psychology and manifesting itself across a number of potentially urge-driven behaviors, including buying, gambling, sexual behaviors, e-mailing, texting, and sexting (Aboujaoude, 2011, 2017). Whiteside and Lynam's (2001) Urgency, Premeditation-Perseverance-Sensationseeking (UPPS) model defines four facets of impulsivity: (a) urgency, or the tendency to experience strong reactions in response to negative (negative urgency) or positive (positive urgency) affect; (b) lack of premeditation, or the difficulty taking into account the consequences of an act before engaging in it; (c) lack of perseverance, or the difficulty remaining focused on a task that may be boring or difficult; and (d) sensation-seeking, or the tendency to pursue exciting new experiences (Cyders \& Smith, 2008). The impulsivity trait has been positively associated with Tinder use in a small sample of 57 users (Carpenter \& McEwan, 2016), but no study has taken a multidimensional approach to impulsivity in relation to adaptive or problematic Tinder use.

\section{Attachment style}

Attachment has been defined as an innate, adaptive system that maintains proximity between infants and caregivers, progressively enabling infants to form stable mental representations of others and ultimately influencing adult relationships, including romantic ones (Ainsworth, 1989). Adult attachment is organized around two main dimensions (Brennan, Clark, \& Shaver, 1998): (a) avoidance, which reflects a tendency to distrust a significant other's goodwill, fear intimacy, and maintain emotional distance; and (b) anxiety, which reflects a tendency to fear that the significant other will not be available in times of need and to fear rejection and abandonment. Avoidant attachment has been associated with lower frequency of sexual intercourse and more uncommitted sexual relationships (BeaulieuPelletier, Philippe, Lecours, \& Couture, 2011), whereas anxious attachment has been related to higher rates of sexual intercourse, especially among women, with the aim to establish closeness and reduce insecurities (Stefanou \& McCabe, 2012). Positive associations have also been reported between anxious and avoidant attachment and compulsive sexual behaviors as well as between avoidant attachment and cybersex addiction (Engel et al., 2019; Varfi et al., 2019; Weinstein, Katz, Eberhardt, Cohen, \& Lejoyeux, 2015). However, no study has examined the possible relationship between attachment style and adaptive or problematic Tinder use. It should also be emphasized that motivations for humans to engage in sexual behaviors are heterogeneous and also involve sexual desire, coping, or social needs (e.g., Ben-Brahim, Rothen, Bianchi De Michelli, Courtois, \& Khazaal, 2019).

\section{Sexual desire}

Sexual desire has been defined as "the sum of the forces that lean us toward and push us away from sexual behavior" (Levine, 2003, p. 280). Sexual desire is not only a determinant of sexual behavior, but is also central to the development of romantic relationships and influences their quality, stability, and the reported couple satisfaction (Bancroft, Graham, Janssen, \& Sanders, 2009; Birnbaum, 2017). However, studies on the association between sexual desire and Tinder use are lacking.

\section{Objectives}

The first aim of the study was to examine via a range of psychological factors - impulsivity, motives for use, attachment style, sexual desire, and self-esteem - whether subgroups of Tinder users can be identified through cluster analysis. Compared to more traditional linear models such as regression, cluster analysis emphasizes the diversity among individuals (Rapkin \& Luke, 1993) and allows the appraisal of complex, non-linear interactions, thereby providing superior ecological validity. Indeed, linear models may omit relationships that are significant for some, but not necessary all, individuals (von Eye \& Bogat, 2006). The second aim was to investigate to what extent these subgroups may differ on various external correlates, including problematic use, depressive mood, use characteristics, and demographic variables.

\section{METHODS}

\section{Subjects and procedure}

Subjects consisted of English-speaking Tinder users aged 18 years or older who were recruited on social networking sites. A total of 1,697 subjects began the survey, of whom 1,159 ( $46.59 \%$ females) completed all questionnaires and reported a heterosexual orientation, and were thus retained in the 
final analysis. Subjects' age ranged from 18 to 74 years $(M=30.02, S D=9.19)$.

\section{Measures}

Problematic Tinder Use Scale (PTUS). The 6-item PTUS (Orosz et al., 2016) was constructed using a six-component addiction model (Griffiths, 2005) that includes salience, tolerance, mood modification, relapse, withdrawal, and conflict. Items are rated on a 5-point Likert scale ranging from 1 (never) to 5 (always), with higher scores suggesting problematic use. The PTUS was found to have good factor structure and moderate internal consistency (Orosz et al., 2016).

Tinder-use patterns. A questionnaire assessed the pattern of Tinder use, including: the number of Tinder-initiated online and offline contacts in the preceding 6 months, ranging from 1 ( 0 person) to 8 (more than 50 persons); looking for committed romantic partners, ranging from 1 (not true at all) to 7 (absolutely true); looking for sexual partners, ranging from 1 (not true at all) to 7 (absolutely true); and the number of current matches indicated on the app.

Short Happiness and Depression Scale (SDHS). The SDHS (Joseph, Linley, Harwood, Lewis, \& McCollam, 2004) consists of six items assessing happiness (e.g., "I feel happy") or depression (e.g., "I feel dissatisfied with my life"). Scale items are scored from 1 (never) to 4 (often). We reversed happiness items so that higher scores reflected depressive mood. The SDHS has shown good internal consistency, test-retest reliability, and convergent and discriminant validity (Joseph et al., 2004).

Cybersex Motives Questionnaire (CMQ). The CMQ (Franc et al., 2018) consists of 14 items that assess three possible motives for cybersex: enhancement, which reflects a motive to increase positive emotions (e.g., "to be entertained"); coping, which reflects strategies that reduce depressive affect (e.g., "to forget my problems"); and social, which reflects a desire to increase social affiliation (e.g., "because I need to socialize with others"). Items are rated on a 5-point Likert scale ranging from 1 (never) to 5 (always or almost always). Higher scores indicate greater endorsement of the motive. This measure has appropriate factor structure and good internal consistency, and positively correlates with sexual desire (Franc et al., 2018). For this study, scale instructions were adapted to address Tinder use only.

Sexual Desire Inventory (SDI). The SDI scale (Spector, Carey, \& Steinberg, 1996) consists of 14 items that assess two dimensions of sexual desire: (a) dyadic (i.e., the desire to have sexual activity with another person) and (b) solitary (i.e., the desire to engage in sexual behavior alone). A Likert scale is used to measure the frequency from 0 (not at all) to 7 (more than once a day), the intensity from 0 (no desire) to 8 (strong desire), and the importance from 0 (not at all important) to 8 (extremely important), of each dimension. Two scores were calculated: one for dyadic and one for solitary sexual desire. The SDI has been shown to have acceptable to excellent internal consistency, good test-retest reliability, and good convergent validity with measures of sexual satisfaction (Mark, Vowels, \& Murray, 2018; Spector et al., 1996).
Experiences in Close Relationships - Revised (ECR-R) questionnaire. The ECR-R scale (Fraley, Waller, \& Brennan, 2000) consists of 36 items that assess two dimensions of adult romantic attachment: (a) avoidance (i.e., discomfort with closeness and dependence on others); and (b) anxiety (i.e., the fear of rejection). Each item is rated on a 7-point scale ranging from 1 (disagree strongly) to 7 (agree strongly). Validation studies have shown high internal reliability, acceptable construct validity, and test-retest stability (Sibley, Fischer, \& Liu, 2005; Sibley \& Liu, 2004).

Short UPPS-P Impulsivity Behavior Scale. The short UPPS-P scale (Billieux et al., 2012) is a 20-item self-report measure that assesses five facets of impulsivity: positive urgency (e.g., "When I'm happy, I often can't stop myself from going overboard"), negative urgency (e.g., "When I feel rejected, I often say things that I later regret"), (lack of) perseverance (e.g., "I am a person who always gets the job done"), (lack of) premeditation (e.g., "I usually make up my mind through careful reasoning"), and sensation-seeking (e.g., "I welcome new and exciting experiences, even if they are a little frightening or unconventional"). Items are rated on a 4-point scale ranging from 1 (I agree strongly) to 4 (I disagree strongly), with higher scores indicating greater impulsivity. This measure has good internal consistency, test-retest stability, and predictive validity (Billieux et al., 2012).

Single-Item Self-Esteem Scale (SISE). This scale consists of one item ("I have high self-esteem") that is rated on a 5-point Likert scale, ranging from 1 (not very true for me) to 5 (very true for me). The SISE scale has shown good convergent validity with other self-esteem measures such as the Rosenberg Self-Esteem Scale (Robins, Hendin, \& Trzesniewski, 2001).

\section{Data analysis}

Following the procedure suggested by Clatworthy, Buick, Hankins, Weinman, and Horne (2005), cluster analyses were used to identify subgroups among Tinder users based on relevant psychological factors (motives, impulsivity, self-esteem, sexual desire, and attachment style). A hierarchical agglomerative method (Ward's method) with squared Euclidian distance was first selected to explore the possible number of clusters. Once the number was defined using dendrogram and agglomeration coefficients, an iterative partitioning clustering method (K-means) was used to maximize similarity within clusters and dissimilarity among clusters. The stability of the cluster structure was examined by determining the agreement between the two methods using Cramer's $V$ test. This test allows determination of whether similar clusters are present regardless of the algorithm used to derive them. In addition, to confirm differences among clusters, scores for each of the variables included in the cluster analyses were entered into discriminant function analysis, where the identified clusters served as the grouping variable. This allows determination of the extent to which clusters are separated by the severity of the Tinder-use variables.

Analyses of variance (ANOVAs) and Kruskall-Wallis tests (for ordinal data or in case assumptions for parametric analyses were violated) were then performed for cluster 
profiling and external correlates (problematic Tinder use, Tinder-use patterns, and depressive mood), followed by Games-Howell or Mann-Whitney $U$ post-hoc tests. In addition, log-linear analyses were used to probe the association between categorical data (gender and relationship status) and cluster membership with $\chi^{2}$ post-hoc tests. Given the large number of analyses, a $p$ value of .001 was used as a cut-off for statistical significance to guard against Type I error. All analyses were two-tailed.

\section{Ethics}

The study is part of a larger study on cybersex. It was carried out in accordance with the Declaration of Helsinki and was approved by the Ethical Committee of the Geneva University Hospital. Subjects received detailed online information about the study. They provided informed consent online and then completed the questionnaires anonymously via SurveyMonkey links. The survey responses were sent over a secure, Secure Socket Layer-encrypted connection.

\section{RESULTS}

\section{Cluster analyses}

All variables included in the cluster analysis were $z$-transformed to share the same metric, so each would contribute equally to cluster formation. Since multicollinearity between variables may impact cluster analysis by giving more weight to collinear variables (Hair, Black, Babin, \& Anderson, 2010), an analysis of correlations among the variables selected for the cluster analysis was performed. The results indicated that the positive and negative urgency subscales of the UPPS-P highly correlated $(r=.60, p<.0001)$, as did the lack of premeditation and lack of perseverance subscales $(r=.51, p<.0001)$. Consequently, these variables were regrouped into a single factor of urgency (Cronbach's $\alpha=.84$ ) and lack of conscientiousness (Cronbach's $\alpha=.84$ ), respectively. Similarly, the three motives of the CMQ highly correlated ( $r$ range: .62-.70; all $p$ 's $<.0001)$ and were regrouped in a general cybersex motives factor (Cronbach's $\alpha=.91$ ). Of note, the distribution of the variables included in the cluster analyses did not strongly deviate from normality, considering that absolute values for skewness and kurtosis greater than 3 and 20, respectively, are judged to be extreme (Weston \& Gore, 2006). Specifically, the results showed that skewness ranged from 0.10 to 0.78 and kurtosis from 0.03 to 0.83 .

The hierarchical cluster analysis was performed using Ward's method with squared Euclidian distance measurement. Visual inspection of the dendrogram and agglomeration coefficients obtained with Ward's method indicated a four-cluster solution. Cluster memberships were then determined through consecutive non-hierarchical K-means cluster analyses computed to identify an optimal fourfactor solution. The four-cluster solution was supported by analyses indicating good agreement between Ward's method and K-means clustering (Cramer's $V=0.63$, $p<.0001)$. The profiles of the four clusters are depicted in Figure 1. Each cluster exceeds $10 \%$ of the sample, as recommended by Hair et al. (2010). Furthermore, discriminant function analysis indicated that the four clusters were adequately separated in discriminant function space (Figure 2) and that, overall, 94\% of the cases were correctly classified in their respective cluster.

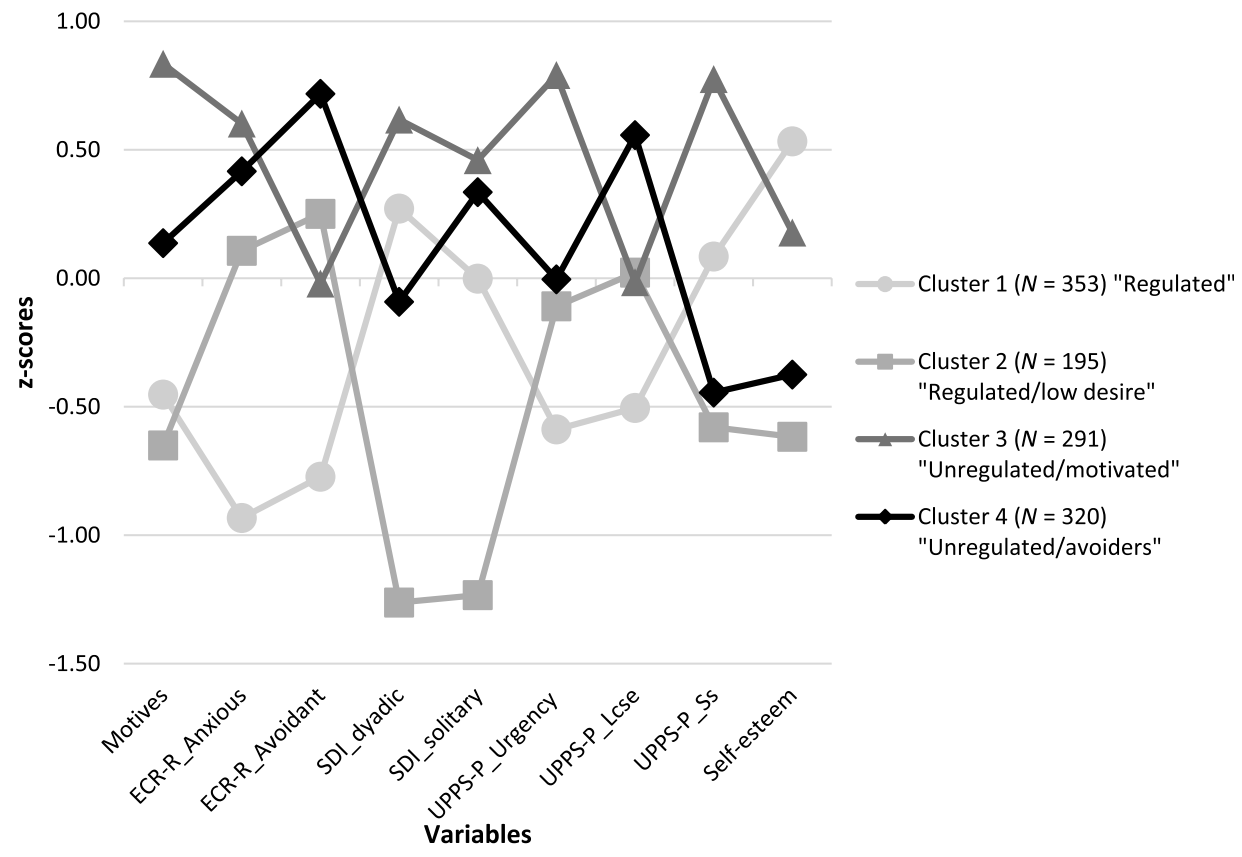

Figure 1. Means for Tinder-use-related factor scores in cluster profiling ( $z$-transformed). Motives: global score from the cyber sex motives questionnaire adapted for Tinder; ECR-R: Experiences in Close Relationships - Revised; SDI: Sexual Desire Inventory; UPPS-P_Urgency: urgency factor from the short version of the UPPS Impulsive Behavior Scale; UPPS-P_Lcse: lack of conscientiousness factor from the short version of the UPPS Impulsive Behavior Scale; UPPS-P_Ss: sensation-seeking factor from the short version of the UPPS Impulsive Behavior Scale; Self-esteem: score from the SISE 


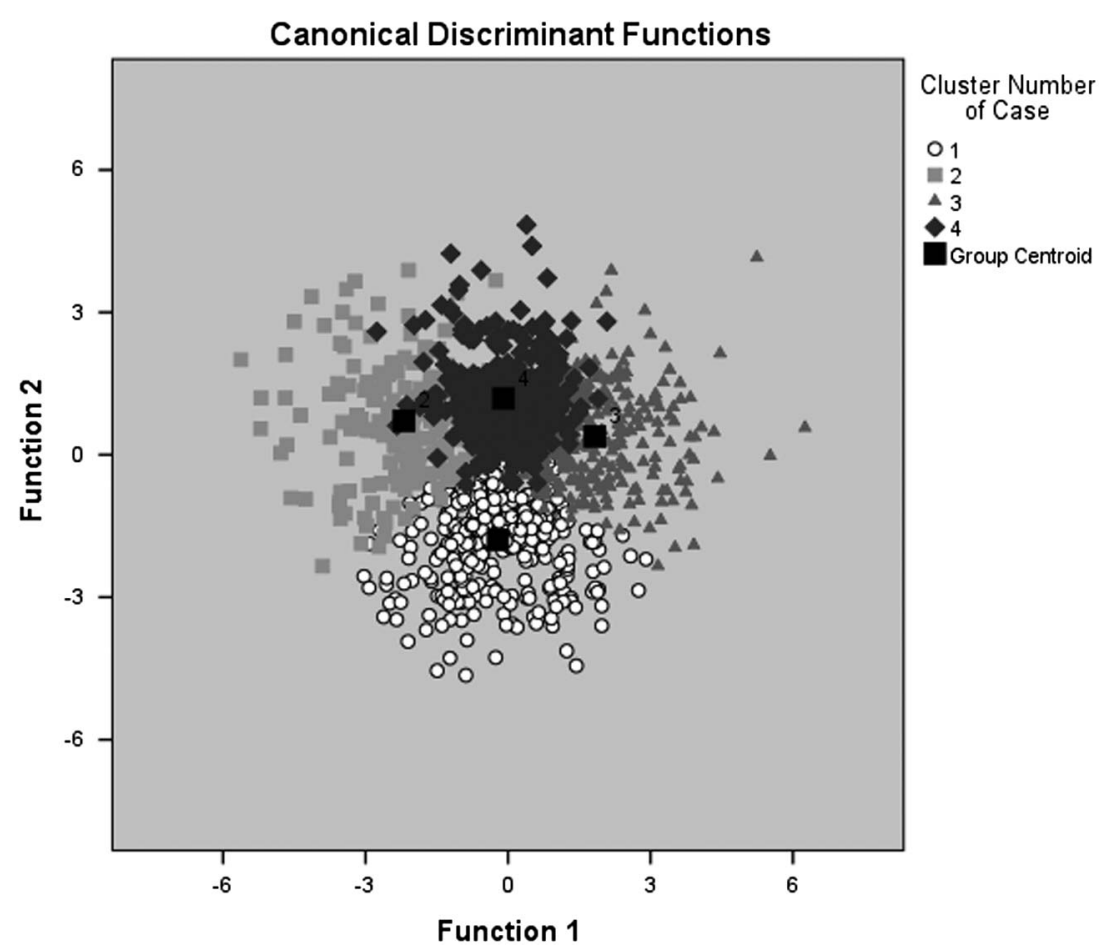

Figure 2. Four-cluster solution plotted in discriminant function space

\section{Group comparisons}

The ANOVAs revealed a significant effect of cluster membership on each psychological factor investigated. Descriptive statistics for each cluster and ANOVAs, including the results of the post-hoc analyses, are shown in Table 1 .

A first cluster (Cluster 1) was characterized by a low level of general motives to use Tinder, secure attachment, a medium level of both dyadic and solitary sexual desire, a low level of both urgency and lack of conscientiousness, a medium level of sensation-seeking, and a high level of selfesteem. This cluster was labeled "regulated" $(N=353$, $30.46 \%$ of the total sample). A second cluster (Cluster 2) was characterized by a low level of general motives to use Tinder, medium insecure attachment, very low levels of both dyadic and solitary sexual desire, a medium level of both urgency and lack of conscientiousness, a low level of sensation-seeking, and low self-esteem. This cluster was labeled "regulated with low desire" $(N=195,16.82 \%$ of the total sample). A third cluster (Cluster 3 ) was characterized by a high level of general motives to use Tinder, a high level of anxious attachment, a medium level of avoidant attachment, a high level of both dyadic and solitary sexual desire, a high level of urgency, a medium level of lack of conscientiousness, a high level of sensation-seeking, and a medium level of selfesteem. This cluster was labeled "unregulated and highly motivated" ( $N=291,25.11 \%$ of the total sample). Finally, a fourth cluster (Cluster 4 ) was characterized by a medium level of motives to use Tinder, a medium level of anxious attachment, a high level of avoidant attachment, a low dyadic sexual desire, a high level of solitary sexual desire, a medium level of urgency, a high level of lack of conscientiousness, a low level of sensation-seeking, and low self-esteem. This cluster was labeled "unregulated avoidant" $(N=320,27.61 \%$ of the total sample).
Regarding external correlates, descriptive statistics for each cluster and group comparisons, including the results of the post-hoc analyses, are shown in Table 2 . The results show that problematic use was greater for Cluster 3 followed by Cluster 4, whereas Clusters 1 and 2 showed significantly lower levels of problematic use. Regarding depressive mood, Cluster 1 reported the lowest level, followed by Clusters 2 and 3, whereas Cluster 4 showed the highest level. As for Tinder-use patterns, subjects in Clusters 3 and 4 were more likely to look for committed partners than those in Clusters 1 and 2. In addition, subjects in Cluster 3 looked significantly more for sexual partners, followed by those from Cluster 1, Cluster 4, and finally Cluster 2. In addition, Cluster 3 subjects reported a significantly greater number of online contacts in the preceding 6 months than those from Cluster 4, Cluster 1, and Cluster 2. Furthermore, Cluster 3 subjects reported more offline contacts, followed by those from Clusters 4 and 1 and, finally, Cluster 2. Finally, Cluster 3 subjects reported the greatest number of current matches, followed by those from Clustesr 1 and 4, whereas Cluster 2 subjects reported the least number of current matches.

Regarding demographics, there was no significant effect based on age. The two-way log-linear analyses examining the association between cluster membership and gender and relationship status, respectively, produced in all cases, a final model that retained all effects including interaction effects. The likelihood ratio of these models was always $\chi^{2}(0)=0, p=1$, indicating that the models fit the data very well (i.e., observed and expected frequences were very similar). The interaction cluster by gender, $\chi^{2}(3)=109.00, p<.0001$, and cluster by relationship status, $\chi^{2}(6)=43.30, p<.001$, were significant. To break down these effects, $\chi^{2}$ post-hoc tests were peformed separately for each cluster. The results indicated that 
Table 1. Descriptive statistics, ANOVAs, and post-hoc tests for cluster profiling

\begin{tabular}{|c|c|c|c|c|c|c|}
\hline \multirow[b]{3}{*}{ Cluster profiling } & $\begin{array}{l}\text { Cluster } 1 \\
(N=353)\end{array}$ & $\begin{array}{l}\text { Cluster } 2 \\
(N=195)\end{array}$ & Cluster $3(N=291)$ & Cluster $4(N=320)$ & \multirow[b]{3}{*}{$F$} & \multirow[b]{3}{*}{$p$} \\
\hline & "Regulated" & $\begin{array}{l}\text { "Regulated/low } \\
\text { desire" }\end{array}$ & $\begin{array}{l}\text { "Unregulated/ } \\
\text { motivated" }\end{array}$ & $\begin{array}{l}\text { "Unregulated/ } \\
\text { avoidant" }\end{array}$ & & \\
\hline & $M(S D)$ & $M(S D)$ & $M(S D)$ & $M(S D)$ & & \\
\hline CMQ_Total motives & $2.15(0.70)^{\mathrm{c}, \mathrm{d}}$ & $1.99(0.72)^{\mathrm{c}, \mathrm{d}}$ & $3.16(0.61)^{\mathrm{a}, \mathrm{b}, \mathrm{d}}$ & $2.61(0.61)^{\mathrm{a}, \mathrm{b}, \mathrm{c}}$ & 179.33 & $<.0001$ \\
\hline ECR-R_Anxious attachment & $2.59(0.91)^{\mathrm{b}, \mathrm{c}, \mathrm{d}}$ & $3.92(1.10)^{\mathrm{a}, \mathrm{c}, \mathrm{d}}$ & $4.55(1.05)^{\mathrm{a}, \mathrm{b}}$ & $4.32(0.93)^{\mathrm{a}, \mathrm{b}}$ & 287.76 & $<.0001$ \\
\hline $\begin{array}{l}\text { ECR-R_Avoidant } \\
\text { attachment }\end{array}$ & $2.38(0.87)^{\mathrm{b}, \mathrm{c}, \mathrm{d}}$ & $3.46(1.04)^{\mathrm{a}, \mathrm{d}}$ & $3.18(0.83)^{\mathrm{a}, \mathrm{d}}$ & $3.96(0.76)^{\mathrm{a}, \mathrm{b}, \mathrm{c}}$ & 212.66 & $<.0001$ \\
\hline SDI_Dyadic sexual desire & $6.16(1.16)^{\mathrm{b}, \mathrm{c}, \mathrm{d}}$ & $3.85(1.51)^{\mathrm{a}, \mathrm{c}, \mathrm{d}}$ & $6.69(0.94)^{\mathrm{a}, \mathrm{b}, \mathrm{d}}$ & $5.62(1.19)^{\mathrm{a}, \mathrm{b}, \mathrm{c}}$ & 197.662 & $<.0001$ \\
\hline SDI_Solitary sexual desire & $4.74(1.86)^{\mathrm{b}, \mathrm{c}, \mathrm{d}}$ & $2.18(1.28)^{\mathrm{a}, \mathrm{c}, \mathrm{d}}$ & $5.70(1.92)^{\mathrm{a}, \mathrm{b}}$ & $5.44(1.49)^{\mathrm{a}, \mathrm{b}}$ & 296.27 & $<.0001$ \\
\hline UPPS-P_Urgency & $2.29(0.48)^{\mathrm{b}, \mathrm{c}, \mathrm{d}}$ & $2.56(0.57)^{\mathrm{a}, \mathrm{c}}$ & $3.07(0.45)^{\mathrm{a}, \mathrm{b}, \mathrm{d}}$ & $2.62(0.46)^{\mathrm{a}, \mathrm{c}}$ & 150.78 & $<.0001$ \\
\hline $\begin{array}{l}\text { UPPS-P_Lack of } \\
\text { conscientiousness }\end{array}$ & $1.66(0.39)^{\mathrm{b}, \mathrm{c}, \mathrm{d}}$ & $1.91(0.44)^{\mathrm{a}, \mathrm{d}}$ & $1.89(0.46)^{\mathrm{a}, \mathrm{d}}$ & $2.16(0.43)^{\mathrm{a}, \mathrm{b}, \mathrm{c}}$ & 82.18 & $<.0001$ \\
\hline UPPS-P_Sensation-seeking & $2.79(0.57)^{\mathrm{b}, \mathrm{c}, \mathrm{d}}$ & $2.38(0.62)^{\mathrm{a}, \mathrm{c}}$ & $3.22(0.47)^{\mathrm{a}, \mathrm{b}, \mathrm{d}}$ & $2.47(0.47)^{\mathrm{a}, \mathrm{c}}$ & 158.67 & $<.0001$ \\
\hline SISE_Self-esteem & $2.86(0.72)^{\mathrm{b}, \mathrm{c}, \mathrm{d}}$ & $1.90(0.75)^{\mathrm{a}, \mathrm{c}}$ & $2.56(0.79)^{\mathrm{a}, \mathrm{b}, \mathrm{d}}$ & $2.10(0.76)^{\mathrm{a}, \mathrm{c}}$ & 98.32 & $<.0001$ \\
\hline
\end{tabular}

Note. Means in the same row that do not share the same superscripts differ at $p<.001$. CMQ: Cybersex motives questionnaire; ECR-R: Experiences in Close Relationships - Revised; SDI: Sexual Desire Inventory; UPPS-P: short version of the UPPS Impulsive Behavior Scale; SISE: Single-Item Self-Esteem Scale. Welch $F$ and Games-Howell post-hoc tests were used because of unequal sample size and because homogeneity of variance assumption was violated in some cases; $S D$ : standard deviation; ANOVAs: analyses of variance.

${ }^{\mathrm{a}}$ Statistically significant in comparison to Cluster 1 . ${ }^{\mathrm{b}}$ Statistically significant in comparison to Cluster 2 . ${ }^{\mathrm{c}}$ Statistically significant in comparison to Cluster $3 .{ }^{\mathrm{d}}$ Statistically significant in comparison to Cluster 4.

Table 2. Descriptive statistics, ANOVAs $(F)$, Kruskall-Wallis $(H)$, and post-hoc tests on external correlates

\begin{tabular}{|c|c|c|c|c|c|c|}
\hline \multirow[b]{3}{*}{ Variable } & \multirow{2}{*}{$\begin{array}{l}\text { Cluster 1 } \\
(N=353) \\
\text { "Regulated" }\end{array}$} & \multirow{2}{*}{$\frac{\text { Cluster } 2(N=195)}{\begin{array}{c}\text { "Regulated/low } \\
\text { desire" }\end{array}}$} & \multirow{2}{*}{ 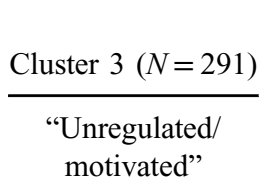 } & \multirow{2}{*}{$\begin{array}{c}\begin{array}{c}\text { Cluster } 4 \\
(N=320)\end{array} \\
\begin{array}{c}\text { "Unregulated/ } \\
\text { avoidant" }\end{array}\end{array}$} & \multirow[b]{3}{*}{$F / H$} & \multirow[b]{3}{*}{$p$} \\
\hline & & & & & & \\
\hline & $M(S D)$ & $M(S D)$ & $M(S D)$ & $M(S D)$ & & \\
\hline PTUS & $1.62(0.50)^{\mathrm{c}, \mathrm{d}}$ & $1.54(0.54)^{\mathrm{c}, \mathrm{d}}$ & $2.40(0.76)^{\mathrm{a}, \mathrm{b}, \mathrm{d}}$ & $2.04(0.65)^{\mathrm{a}, \mathrm{b}, \mathrm{c}}$ & 105.47 & $<.0001$ \\
\hline SDHS_Depressive mood & $1.77(0.55)^{\mathrm{b}, \mathrm{c}, \mathrm{d}}$ & $2.42(0.64)^{\mathrm{a}}$ & $2.21(0.60)^{\mathrm{a}, \mathrm{d}}$ & $2.48(0.58)^{\mathrm{a}, \mathrm{c}}$ & 101.68 & $<.0001$ \\
\hline \multicolumn{7}{|l|}{ Tinder-use patterns } \\
\hline Looking for committed partners & $2.92(1.93)^{\mathrm{c}, \mathrm{d}}$ & $2.76(1.99)^{\mathrm{c}, \mathrm{d}}$ & $3.70(1.92)^{\mathrm{a}, \mathrm{b}}$ & $3.42(1.80)^{\mathrm{a}, \mathrm{b}}$ & 50.07 & $<.0001$ \\
\hline Looking for sexual partners & $3.08(2.07)^{b, c, d}$ & $2.01(1.38)^{\mathrm{a}, \mathrm{c}, \mathrm{d}}$ & $4.41(1.92)^{\mathrm{a}, \mathrm{b}, \mathrm{d}}$ & $3.80(1.85)^{\mathrm{a}, \mathrm{b}, \mathrm{c}}$ & 190.07 & $<.0001$ \\
\hline $\begin{array}{l}\text { Number of contacts online } \\
\text { during past } 6 \text { months }\end{array}$ & $3.12(1.91)^{\mathrm{c}}$ & $2.61(1.70)^{\mathrm{c}, \mathrm{d}}$ & $4.05(1.82)^{\mathrm{a}, \mathrm{b}, \mathrm{d}}$ & $3.40(1.69)^{\mathrm{b}, \mathrm{c}}$ & 77.07 & $<.0001$ \\
\hline $\begin{array}{l}\text { Number of contacts offline } \\
\text { during past } 6 \text { months }\end{array}$ & $1.85(1.24)^{\mathrm{b}, \mathrm{c}}$ & $1.50(0.94)^{\mathrm{a}, \mathrm{c}, \mathrm{d}}$ & $2.56(1.41)^{\mathrm{a}, \mathrm{b}, \mathrm{d}}$ & $1.99(1.20)^{\mathrm{b}, \mathrm{c}}$ & 101.79 & $<.0001$ \\
\hline Number of current matches & $29.34(107.02)^{\mathrm{c}}$ & $30.35(131.85)^{\mathrm{c}, \mathrm{d}}$ & $51.32(139.29)^{\mathrm{a}, \mathrm{b}, \mathrm{d}}$ & $33.45(100.73)^{b, c}$ & 63.61 & $<.0001$ \\
\hline \multicolumn{7}{|l|}{ Demographic } \\
\hline Age & $30.01(8.80)$ & $31.44(11.36)$ & $29.72(8.14)$ & $29.44(9.03)$ & 1.49 & .22 \\
\hline Men/women (\%) & $33 / 27$ & $6 / 29 * *$ & $29 / 21$ & $32 / 23 * *$ & - & - \\
\hline \multicolumn{7}{|l|}{ Relationship status } \\
\hline Single $(\%)$ & $23 * *$ & 20 & 22 & $35^{* *}$ & - & - \\
\hline In a relationship, not married $(\%)$ & 33 & 13 & 26 & 28 & - & - \\
\hline In a relationship, married (\%) & 37 & 19 & 29 & $15^{* *}$ & - & - \\
\hline
\end{tabular}

Note. Numbers in the same row that do not share the same superscripts differ at $p<.001$. PTUS: Problematic Tinder Use Scale; SDHS: Short Happiness and Depression Scale; ANOVAs: analyses of variance; $S D$ : standard deviation.

${ }^{a}$ Statistically significant in comparison to Cluster $1 .{ }^{\mathrm{b}}$ Statistically significant in comparison to Cluster 2 . ${ }^{\mathrm{c}}$ Statistically significant in comparison to Cluster 3 . ${ }^{\mathrm{d}}$ Statistically significant in comparison to Cluster 4.

*All Games-Howell or Mann-Whitney $U$ post-hoc difference tests are significant with $p<.001$.

women were overrepresented in Cluster $2, \chi^{2}(1)=108.41$, $p<.0001$, whereas men formed a larger proportion of Cluster $4, \chi^{2}(1)=10.92, p<.001$. By contrast, gender was not significantly associated with Cluster $1, \chi^{2}(1)=5.58$, $p=.02$, and failed to reach significance for Cluster 3, $\chi^{2}(1)=9.41, p=.002$.

Regarding relationship status, $\chi^{2}$ post-hoc tests indicated that single subjects were less represented in Cluster 1, 
$\chi^{2}(1)=15.47, p<.001$ and more represented in Cluster 4, $\chi^{2}(1)=16.58, p<.001$. In addition, married subjects were less represented in Cluster $4, \chi^{2}(1)=24.25, p<.001$. No other post-hoc tests reached statistical significance.

\section{DISCUSSION}

Digital tools have dramatically changed romantic and mating rituals, with potentially serious sociocultural consequences (Aboujaoude, 2011). This study aimed to determine whether reliable psychological subtypes can be identified among users of Tinder, a leading dating app, and whether those differed on external correlates such as problematic use, depressive mood, Tinder-use patterns, and demographic variables. Four clusters were identified: two with low scores on the PTUS (Clusters 1 and 2), one with an intermediate score (Cluster 4), and one with a high score (Cluster 3).

Cluster 1 ("regulated"), the largest cluster, was characterized by low impulsivity, high self-esteem, secure attachment, medium to high dyadic sexual desire, low depressive mood, and low problematic use. Cluster 1 subjects consider the coping, enhancement, and social motives for using Tinder to be likely secondary. Nevertheless, they reported interest in both committed and casual sexual partners, although to a lesser extent than Clusters 3 and 4. They also had similar numbers of online and offline contacts as well as matches as Cluster 4, but less online and offline contacts than Cluster 3. Cluster 1 subjects may be influenced by other motives not addressed by the CMQ, such as curiosity and distraction, which have also been shown to promote Tinder use (Timmermans \& De Caluwé, 2017).

Cluster 2 ("regulated with low desire"), the smallest cluster, was characterized by low to medium impulsivity, low self-esteem, anxious attachment, low motives, very low dyadic and solitary sexual desire, more depressive mood, and low problematic use. Cluster 2 subjects reported less interest in both committed and casual sexual partners, had less online and offline contacts, and had less current matches. One may hypothesize that, according to them, Tinder is not used as a dating app per se, but perhaps a means to increase self-worth, such as via reassurance around appearance. Alternatively, subjects in this cluster may approach Tinder with depressionlinked helplessness, without any hope of finding something that meets their needs.

Cluster 3 ("unregulated and highly motivated") was characterized by a high level of motives to use Tinder, a high level of anxious attachment, a high level of both dyadic and solitary sexual desire, a high level of urgency and sensation-seeking, and a moderate level of self-esteem. In contrast to Cluster 2, Cluster 3 subjects were strongly interested in looking for both committed and casual sexual partners and were more likely to have online or offline contacts. This cluster had the highest level of problematic use and the greatest number of current matches, while reporting an intermediate level of depressive mood. Cluster 3 subjects' Tinder use was driven by a combination of a high level of motives (social, enhancement, and coping) and poor self-control - in particular urgency, which has been associated with weak inhibitory control and poor decisionmaking (Rochat, Billieux, Gagnon, \& Van der Linden, 2018).
They also have trouble overcoming the immediate gratification provided by the app (e.g., excitement associated with potential sexual activity and reinforcement resulting from frequent offline contacts), as indicated by their high sensation-seeking levels. Accordingly, users with diminished self-control seem to be at higher risk for developing problematic Tinder use, perhaps because of an interaction between poor self-control and strong motives incentivizing app use. It has also been hypothesized that individuals with high urgency engage in behaviors aimed at relieving negative affects (or increasing positives ones) in the short term, despite negative delayed consequences (Selby, Anestis, \& Joiner, 2008). As such, it may be the case that individuals with high urgency are more prone to uncontrolled Tinder use, and that this activity constitutes dysfunctional coping aimed at relieving or suppressing adverse affects or increasing positive ones. In this context, app use may interact with psychological discomfort linked to subjects' moderate levels of depressive mood and anxious attachment, for instance when they attempt to regulate distress following app interactions. The availability of many potential mates in their geographic vicinity may thus compensate for their fear of rejection and abandonment inasmuch as they can quickly seek approval and reassurance from other potential partners.

Finally, Cluster 4 ("unregulated avoidant") was characterized by a medium level of motives to use Tinder, a high level of avoidant attachment, a high level of solitary sexual desire, low conscientiousness, low sensation-seeking, and low self-esteem. These subjects reported the highest level of depressive mood and the second highest level of problematic use after Cluster 3 . In addition, they are equally prone to look for committed partners as subjects in Cluster 3. However, they reported looking for sexual partners less than Cluster 3, but more than Clusters 1 and 2. They also reported the second highest number of online and offline contacts after Cluster 3 subjects. These subjects, who had low self-esteem and a high level of avoidant attachment, might be more inclined to use social networking sites because they may view online environments as safer to express themselves or to meet potential partners. The greater number of single subjects in this cluster suggests that they struggle with meeting partners or maintaining relationships in real life. According to them, Tinder use may not be threatening with respect to their discomfort with closeness or dependence on others (Blackhart, Fitzpatrick, \& Williamson, 2014) inasmuch as the app could satisfy a need for social or sexual communication without spatial (e.g., proximity) and social (e.g., possibility to unilaterally swipe left on a potential match without consequences) constraints (Sumter et al., 2017; Valkenburg \& Peter, 2007). This subgroup is also characterized by low conscientiousness, which has been previously related to using Tinder to alleviate boredom (Orosz et al., 2018). Finally, these subjects reported a high level of solitary sexual desire. One may hypothesize that solitary sexuality, potentially like Tinder use, is a coping strategy to deal with depressive affect or sexual frustration (Dosch, Rochat, Ghisletta, Favez, \& Van der Linden, 2016). Further studies are needed to assess this hypothesis.

Some limitations to the study must be acknowledged. First, the study is cross-sectional, and further research is 
required to longitudinally confirm the validity of the clusters highlighted. Furthermore, the sample is self-selected, limiting the generalizability of results to the entire population of Tinder users (Khazaal et al., 2014). Moreover, the study relies exclusively on self-reports, and those have been associated with various biases (e.g., social desirability and lack of insight). The sample was also composed entirely of heterosexual-identifying subjects, limiting the applicability of results to non-heterosexual participants. Finally, the number of Tinder-initiated contacts refers to the actual number of partners that users claimed to have interacted with (whether on- or offline). This number may be high in individuals who are "successful" at Tinder, but does not reflect individuals who compulsively use the platform (e.g., by right-swiping a lot) but fail to get matches. Further studies should thus focus on the determinants of successful versus unsuccessful Tinder use.

In conclusion, this is the first study to demonstrate distinct subtypes among Tinder app users. Corroborating previous work on Internet gaming disorder (Billieux et al., 2015), our results suggest that problematic Tinder use is heterogeneous and involves a wide range of interacting psychological factors. Further studies are required to disentangle the role of these factors in the development, perpetuation, and recurrence of problematic use, and to understand the very socially consequential draw that this and similar dating apps have. In addition, some authors have underscored the need to take into account the contextual factors in which problematic online behaviors emerge (Starcevic \& Aboujaoude, 2015). The fact that $22 \%$ of users were married and $44 \%$ were involved in a relationship suggests that poor satisfaction with current relationships may constitute a relevant external factor to consider. Alternatively, the availability of seemingly endless meeting opportunities online may magnify dissatisfaction with relationships and discourage couples therapy or other helpful interventions, sending individuals to dating apps, and sites for quick fixes to relatively minor relationship troubles (Aboujaoude, 2011). It is also possible that some married or coupled participants are not necessarily unsatisfied with their relationships but are involved in sexually open ones, thereby also promoting Tinder use. Finally, the PTUS used in the study is based on the component model of addiction, so that an elevated score on this scale could imply that the person presents an addictive pattern of Tinder use. In this context, excessive Tinder use could be considered a behavioral addiction - whether a distinct one or a variant of sexbased addiction or Internet addiction. It could, indeed, also conceivably meet criteria for an impulse control disorder. However, recent conceptualizations about technologyrelated psychological problems have emphasized the need to avoid overpathologizing everyday behaviors and to focus instead on functional impairment as the critical dimension (Kardefelt-Winther et al., 2017; Starcevic \& Aboujaoude, 2017). Therefore, further studies should determine whether problematic Tinder use actually leads to longterm significant harm by compromising crucial areas of functioning. Still, and although criteria for PTUS remain to be established to identify problematic users, it can be said that, for the majority of subjects, Tinder did not seem to be associated with problematic use: $47 \%$ reported a low score and $27 \%$ an intermediate score. By helping to initiate committed or casual relationships, promoting self-validation, and encouraging socializing, Tinder may be beneficial to many. As such, this app should not be considered a mere "hook-up" tool; it may serve crucial adaptive functions as well.

Funding sources: No financial support was received for this study.

Authors' contribution: YK and FB-D designed the study. YK acquired the data. LR and YK ran the statistical analyses and wrote the initial draft of the article. LR, YK, FB-D, and EA interpreted the results. FB-D and EA reviewed the initial draft and participated in the writing of the final draft. All authors approved the final version of the manuscript.

Conflict of interest: The authors declare no conflict of interest.

\section{REFERENCES}

Aboujaoude, E. (2011). Virtually you: The dangerous powers of the e-personality. New York, NY: W. W. Norton.

Aboujaoude, E. (2017). The Internet's effect on personality traits: An important casualty of the "Internet addiction" paradigm. Journal of Behavioral Addictions, 6(1), 1-4. doi:10.1556/ 2006.6.2017.009

Ainsworth, M. S. (1989). Attachments beyond infancy. American Psychologist, 44(4), 709-716. doi:10.1037/0003-066X.44.4.709

Andreassen, C. S., Pallesen, S., Griffiths, M. D., Torsheim, T., \& Sinha, R. (2018). The development and validation of the Bergen-Yale Sex Addiction Scale with a large national sample. Frontiers in Psychology, 9, 144. doi:10.3389/fpsyg.2018.00144

Bancroft, J., Graham, C. A., Janssen, E., \& Sanders, S. A. (2009). The dual control model: Current status and future directions. Journal of Sex Research, 46(2-3), 121-142. doi:10.1080/00224490 902747222

Beaulieu-Pelletier, G., Philippe, F. L., Lecours, S., \& Couture, S. (2011). The role of attachment avoidance in extradyadic sex. Attachment \& Human Development, 13(3), 293-313. doi:10.1080/14616734.2011.562419

Ben-Brahim, F., Rothen, S., Bianchi De Michelli, F., Courtois, R., \& Khazaal, Y. (2019). Contribution of sexual desire and motives to the compulsive use of cybersex. Journal of Behavioral Addictions, 8(3), 442-450. doi:10.1556/2006.8.2019.47

Billieux, J., Rochat, L., Ceschi, G., Carré, A., Offerlin-Meyer, I., Defeldre, A.-C., Khazaal, Y., Besche-Richard, C., \& Van der Linden, M. (2012). Validation of a short French version of the UPPS-P Impulsive Behavior Scale. Comprehensive Psychiatry, 53(5), 609-615. doi:10.1016/j.comppsych.2011.09.001

Billieux, J., Thorens, G., Khazaal, Y., Zullino, D., Achab, S., \& Van der Linden, M. (2015). Problematic involvement in online games: A cluster analytic approach. Computers in Human Behavior, 43, 242-250. doi:10.1016/j.chb.2014.10.055

Birnbaum, G. E. (2017). The fragile spell of desire: A functional perspective on changes in sexual desire across relationship 
development. Personality and Social Psychology Review, 22(2), 101-127. doi:10.1177/1088868317715350

Blackhart, G. C., Fitzpatrick, J., \& Williamson, J. (2014). Dispositional factors predicting use of online dating sites and behaviors related to online dating. Computers in Human Behavior, 33, 113-118. doi:10.1016/j.chb.2014.01.022

Brennan, K. A., Clark, C. L., \& Shaver, P. R. (1998). Self-report measurement of adult attachment: An integrative overview. In J. A. Simpson \& W. S. Rholes (Eds.), Attachment theory and close relationships (pp. 46-76). New York, NY: Guilford Press.

Carpenter, C. J., \& McEwan, B. (2016, April). The players of micro-dating: Individual and gender differences in goal orientations toward micro-dating apps. First Monday. Retrieved March 29, 2019, from https://uncommonculture.org/ojs/ index.php/fm/article/view/6187/5469

Clatworthy, J., Buick, D., Hankins, M., Weinman, J., \& Horne, R. (2005). The use and reporting of cluster analysis in health psychology: A review. British Journal of Health Psychology, 10(3), 329-358. doi:10.1348/135910705X25697

Cyders, M. A., \& Smith, G. T. (2008). Emotion-based dispositions to rash action: Positive and negative urgency. Psychological Bulletin, 134(6), 807-828. doi:10.1037/a0013341

Daruna, J. H., \& Barnes, P. A. (1993). A neurodevelopmental view of impulsivity. In W. G. McCown, J. L. Johnson, \& M. B. Shure (Eds.), The impulsive client: Theory, research, and treatment (pp. 23-38). Washington, DC: American Psychological Association.

Dosch, A., Rochat, L., Ghisletta, P., Favez, N., \& Van der Linden, M. (2016). Psychological factors involved in sexual desire, sexual activity, and sexual satisfaction: A multi-factorial perspective. Archives of Sexual Behavior, 45(8), 2029-2045. doi:10.1007/s10508-014-0467-z

Engel, J., Veit, M., Sinke, C., Heitland, I., Kneer, J., Hillemacher, T., Hartmann, U., \& Kruger, T. H. C. (2019). Same same but different: A clinical characterization of men with hypersexual disorder in the Sex@Brain Study. Journal of Clinical Medicine, 8(2), 157. doi:10.3390/jcm8020157

Fraley, R. C., Waller, N. G., \& Brennan, K. A. (2000). An item response theory analysis of self-report measures of adult attachment. Journal of Personality and Social Psychology, 78(2), 350-365. doi:10.1037/0022-3514.78.2.350

Franc, E., Khazaal, Y., Jasiowka, K., Lepers, T., BianchiDemicheli, F., \& Rothen, S. (2018). Factor structure of the Cybersex Motives Questionnaire. Journal of Behavioral Addictions, 7(3), 601-609. doi:10.1556/2006.7.2018.67

Gatter, K., \& Hodkinson, K. (2016). On the differences between Tinder $^{\mathrm{TM}}$ versus online dating agencies: Questioning a myth. An exploratory study. Cogent Psychology, 3(1), 1162414. doi:10.1080/23311908.2016.1162414

Griffiths, M. (2005). A 'components' model of addiction within a biopsychosocial framework. Journal of Substance Use, 10(4), 191-197. doi:10.1080/14659890500114359

Hair, J. F., Black, W. C., Babin, B. J., \& Anderson, R. E. (2010). Multivariate data analysis: A global perspective. London, UK: Pearson Education.

Joseph, S., Linley, P. A., Harwood, J., Lewis, C. A., \& McCollam, P. (2004). Rapid assessment of well-being: The Short Depression-Happiness Scale (SDHS). Psychology and Psychotherapy: Theory, Research and Practice, 77(4), 463-478. doi:10.1348/1476083042555406
Kardefelt-Winther, D., Heeren, A., Schimmenti, A., van Rooij, A., Maurage, P., Carras, M., Edman, J., Blaszczynski, A., Khazaal, Y., \& Billieux, J. (2017). How can we conceptualize behavioural addiction without pathologizing common behaviours? Addiction, 112(10), 1709-1715. doi:10.1111/add.13763

Khazaal, Y., van Singer, M., Chatton, A., Achab, S., Zullino, D., Rothen, S., Khan, R., Billieux, J., \& Thorens, G. (2014). Does self-selection affect samples' representativeness in online surveys? An investigation in online video game research. Journal of Medical Internet Research, 16(7), e164. doi:10.2196/ jmir.2759

Laier, C., \& Brand, M. (2014). Empirical evidence and theoretical considerations on factors contributing to cybersex addiction from a cognitive-behavioral view. Sexual Addiction \& Compulsivity, 21(4), 305-321. doi:10.1080/10720162.2014. 970722

Levine, S. B. (2003). The nature of sexual desire: A clinician's perspective. Archives of Sexual Behavior, 32(3), 279-285. doi:10.1023/A:1023421819465

Mark, K. P., Vowels, L. M., \& Murray, S. H. (2018). The impact of attachment style on sexual satisfaction and sexual desire in a sexually diverse sample. Journal of Sex \& Marital Therapy, 44(5), 450-458. doi:10.1080/0092623X.2017.1405310

Orosz, G., Benyó, M., Berkes, B., Nikoletti, E., Gál, É., TóthKirály, I., \& Böthe, B. (2018). The personality, motivational, and need-based background of problematic Tinder use. Journal of Behavioral Addictions, 7(2), 301-316. doi:10.1556/2006. 7.2018.21

Orosz, G., Tóth-Király, I., Bőthe, B., \& Melher, D. (2016). Too many swipes for today: The development of the Problematic Tinder Use Scale (PTUS). Journal of Behavioral Addictions, 5(3), 518-523. doi:10.1556/2006.5.2016.016

Ranzini, G., \& Lutz, C. (2017). Love at first swipe? Explaining Tinder self-presentation and motives. Mobile Media \& Communication, 5(1), 80-101. doi:10.1177/2050157916664559

Rapkin, B. D., \& Luke, D. A. (1993). Cluster analysis in community research: Epistemology and practice. American Journal of Community Psychology, 21(2), 247-277. doi:10.1007/BF00941623

Robins, R. W., Hendin, H. M., \& Trzesniewski, K. H. (2001). Measuring global self-esteem: Construct validation of a single-item measure and the Rosenberg Self-Esteem Scale. Personality and Social Psychology Bulletin, 27(2), 151-161. doi:10.1177/0146167201272002

Rochat, L., Billieux, J., Gagnon, J., \& Van der Linden, M. (2018). A multifactorial and integrative approach to impulsivity in neuropsychology: Insights from the UPPS model of impulsivity. Journal of Clinical and Experimental Neuropsychology, 40(1), 45-61. doi:10.1080/13803395.2017.1313393

Rosenberg, M. (1965). Society and the adolescent self-image. Princeton, NJ: Princeton University Press.

Schrock, A. R. (2015). Communicative affordances of mobile media: Portability, availability, locatability, and multimediality. International Journal of Communication, 9, 1229-1246.

Selby, E. A., Anestis, M. D., \& Joiner, T. E. (2008). Understanding the relationship between emotional and behavioral dysregulation: Emotional cascades. Behaviour Research and Therapy, 46(5), 593-611. doi:10.1016/j.brat.2008.02.002

Sibley, C. G., Fischer, R., \& Liu, J. H. (2005). Reliability and validity of the Revised Experiences in Close Relationships (ECR-R) self-report measure of adult romantic attachment. 
Personality and Social Psychology Bulletin, 31(11), 1524-1536. doi:10.1177/0146167205276865

Sibley, C. G., \& Liu, J. H. (2004). Short-term temporal stability and factor structure of the Revised Experiences in Close Relationships (ECR-R) measure of adult attachment. Personality and Individual Differences, 36(4), 969-975. doi:10.1016/ S0191-8869(03)00165-X

Smith, C. (2018, December). 50 interesting Tinder statistics and facts. Digital Marketing Stats/Strategy/Gadgets. Retrieved March 29, 2019, from https://expandedramblings.com/index. php/tinder-statistics/

Spector, I. P., Carey, M. P., \& Steinberg, L. (1996). The Sexual Desire Inventory: Development, factor structure, and evidence of reliability. Journal of Sex \& Marital Therapy, 22(3), 175-190. doi:10.1080/00926239608414655

Starcevic, V., \& Aboujaoude, E. (2015). Cyberchondria, cyberbullying, cybersuicide, cybersex: New psychopathologies for the 21st century? World Psychiatry, 14(1), 97-100. doi:10.1002/wps.20195

Starcevic, V., \& Aboujaoude, E. (2017). Internet addiction: Reappraisal of an increasingly inadequate concept. CNS Spectrums, 22(1), 7-13. doi:10.1017/S1092852915000863

Stefanou, C., \& McCabe, M. P. (2012). Adult attachment and sexual functioning: A review of past research. The Journal of Sexual Medicine, 9(10), 2499-2507. doi:10.1111/j.17436109.2012.02843.x

Strubel, J., \& Petrie, T. A. (2017). Love me Tinder: Body image and psychosocial functioning among men and women. Body Image, 21, 34-38. doi:10.1016/j.bodyim.2017.02.006

Sumter, S. R., Vandenbosch, L., \& Ligtenberg, L. (2017). Love me Tinder: Untangling emerging adults' motivations for using the dating application Tinder. Telematics and Informatics, 34(1), 67-78. doi:10.1016/j.tele.2016.04.009

Timmermans, E., \& De Caluwé, E. (2017). Development and validation of the Tinder Motives Scale (TMS). Computers in Human Behavior, 70, 341-350. doi:10.1016/j.chb.2017.01.028

Valkenburg, P. M., \& Peter, J. (2007). Who visits online dating sites? Exploring some characteristics of online daters. CyberPsychology \& Behavior, 10(6), 849-852. doi:10.1089/cpb.2007.9941

Varfi, N., Rothen, S., Jasiowka, K., Lepers, T., Bianchi-Demicheli, F., \& Khazaal, Y. (2019). Sexual desire, mood, attachment style, impulsivity, and self-esteem as predictive factors for addictive cybersex. JMIR Mental Health, 6(1), e9978. doi:10.2196/ mental.9978

von Eye, A., \& Bogat, G. A. (2006). Person-oriented and variableoriented research: Concepts, results, and development. MerrillPalmer Quarterly, 52(3), 390-420. doi:10.1353/mpq.2006.0032

Weinstein, A., Katz, L., Eberhardt, H., Cohen, K., \& Lejoyeux, M. (2015). Sexual compulsion-relationship with sex, attachment and sexual orientation. Journal of Behavioral Addictions, 4(1), 22-26. doi:10.1556/JBA.4.2015.1.6

Weston, R., \& Gore, P. A. (2006). A brief guide to structural equation modeling. The Counseling Psychologist, 34(5), 719-751. doi:10.1177/0011000006286345

Whiteside, S. P., \& Lynam, D. R. (2001). The five factor model and impulsivity: Using a structural model of personality to understand impulsivity. Personality and Individual Differences, 30(4), 669-689. doi:10.1016/S0191-8869(00)00064-7

Ybarra, M. L., \& Mitchell, K. J. (2014). "Sexting” and its relation to sexual activity and sexual risk behavior in a national survey of adolescents. Journal of Adolescent Health, 55(6), 757-764. doi:10.1016/j.jadohealth.2014.07.012 